INTERNATIONAL JOURNAL OF MULTidisciplinARY RESEARCH AND ANALYSis

ISSN(print): 2643-9840, ISSN(online): 2643-9875

Volume 05 Issue 02 February 2022

DOI: 10.47191/ijmra/v5-i2-02, Impact Factor: 6.072

Page No.- 256-262

\title{
Impact Evaluation of The Internal Control System on The Sales Function Performance Within Moroccan Companies' Context
}

\author{
Abdelali BABAALI ${ }^{1}$, Fatima Zahra ACHOUR ${ }^{2}$ \\ ${ }^{1,2}$ Department, Ibn Tofaïl University, Kenitra, Morocco
}

\begin{abstract}
Moroccan business leaders are increasingly concerned about the phenomenon of declining commercial activity, confirmed by the economic barometers published by the General Confederation of Moroccan Enterprises (CGEM) during the last four years (2015-2018), and the direct causes that might be linked to unfair competition, payment delays problem, unpaid debts and finally the widespread of a heavy informal sector. Through this article, we are going to focus on the relationship that may exist between the components of an internal control system setup in a sales function and its performance. In other words, we are looking to explain the decreasing performance of the sales function using the evaluation of the internal control system deployed there. To do this, we will present in the first part, the theoretical foundations of the internal control system and socio-economic performance. Then, we will analyze the points of connection and influence to finally develop some hypotheses, which will be the subject of an empirical study based on of one hundred and sixty-one Moroccan companies. The results of our work provide an answer on the degree of influence of internal control system components of the commercial function on its socio-economic performance and more precisely on its commercial performance.
\end{abstract}

KEYWORDS: Internal control system, socio-economic performance, commercial performance COSO standard.

\section{INTRODUCTION}

In a context of economic openness, and on the basis of studies carried out by the CGEM [1] and more precisely in its reports published under the name 'economic barometer', a large number of Moroccan business leaders expressed, during the four in recent years, a poor perception mainly due to the non-performance of their companies observed in particular at the level of their commercial functions. The causes put forward by the business leaders interviewed during the studies carried out can be summed up in the decline in economic activity, unfair competition, the problem of payment delays, unpaid debts and finally the existence of a heavy informal sector.

The problem of the non-performance of the commercial functions of Moroccan companies, manifested by the causes cited below, aroused our curiosity as a researcher and led us to look into the reasons that could possibly explain the contribution of an internal control implemented within a sales function to improve its performance. To do this, we will first ask ourselves the following questions:

Are Moroccan companies concerned about the concern of controlling activities at the level of their commercial functions?

Do Moroccan companies ensure that their commercial functions are endowed with an internal control system which is specific to them and which participates in the control of all activities?

To our knowledge and on the basis of the research carried out, there is no scientific work explaining the performance of the commercial function of Moroccan companies by the effectiveness of the internal control system established there.

According to an institutionalist approach, and in a rapidly changing open economy, the poor performance of the commercial function within Moroccan companies could then be explained by the absence of an effective internal control system or the existence of an irrelevant internal control.

This article aims to answer the following question:

To what extent the internal control system influences the performance of the sales function within companies that have recorded a decline in activity and operate in a context of unfair competition, the problem of payment deadlines and unpaid debts and a heavy informal sector? 


\section{Impact Evaluation of The Internal Control System on The Sales Function Performance Within Moroccan Companies' Context}

Based on our research problem and under the economic conditions mentioned above, our main objective, through this contribution, is to understand the impact of the implementation of an internal control system at the level of the commercial function on its performance.

To do this, we will try to organize the work as follows:

First of all, in the first part, we will present the theoretical foundations of the internal control system and that of organizational performance by referring to works to give general definitions.

Also, we will try to mobilize a theory to develop a conceptual definition that will help us easily extract the hypotheses to finally conclude with a theoretical model of this research work.

Then, in a second part, we will try to identify the different variables of an internal control system, and this by using the empirical work of previous research to finally design our conceptual model.

Then, in a third part, we will examine the influence of the different components of an internal control system of a commercial function by a quantitative study on a sample of 161 Moroccan companies.

Finally, we will present the results obtained with a detailed discussion of the answer to our problem.

\section{THE INTERNAL CONTROL SYSTEM: HISTORY OF THE MAIN DEFINITIONS AND NEW ROLE}

Several definitions, developed by different bodies and committees, have been reported in the majority of scientific research work on the subject of internal control. In the present work, we have tried to select the most widely answered definitions and we have classified them in chronological order to highlight a history that traces the evolution of this concept since its appearance.

The first definition of internal control having been a real success due to the fact that it underlined a real overstepping of the usual accounting framework which was based solely on financial verification is the work of the French Order of Chartered Accountants in 1977. The latter shared a broader vision of internal control by defining it as follows: "Internal control is the set of securities contributing to the control of the company. It aims on the one hand to ensure the protection and safeguard of the heritage and the quality of the information, on the other to apply the instructions of the management and to promote the improvement of performances. It manifests itself in the organization, methods and procedures of each of the company's activities, to maintain its sustainability. "(Renard, 2012) [2]

The second famous definition, dating from 1992, is established by The Committee Of Sponsoring Organizations of the Treadway Commission. In fact, it is a non-profit commission that has established a standard definition of internal control and created a framework to assess its effectiveness. By extension, this standard is also called coso. For this commission "internal control is a process implemented by the board of directors, managers and staff of an organization intended to provide reasonable assurance as to the achievement of objectives".

In fact, the particular contribution of this definition is that it involves all the resources of the company from the base of the organization to its top. Each employee, from his hierarchical level, has his own internal control to monitor and respect in order to converge on the control of activities and the achievement of the objectives set.

The third definition was devised in 1995 by the Canadian Institute of Chartered Accountants. This institute describes control as "elements of the organization (including resources, systems, processes, culture and tasks) which, taken together, help to achieve objectives". It should be noted that the particularity of this definition is that it has placed the emphasis on the medium component and therefore it complements that of the coso.

In addition to the three definitions cited above, we add the definition reported in the internal control guide (report published in 1999), known under the name "the Turnbull guidance" which takes up most of the coso and Coco definitions in them. Supplementing with objectives. According to this guide, internal control as a system that encompasses policies, processes, tasks, behaviors and other aspects that are combined together in order to:

- Facilitate the effectiveness and efficiency of operations;

- Help ensure the quality of internal and external reporting;

- And help ensure compliance with laws and regulations.

In 2006, the French markets authority challenged internal control by using basic definitions as "a company device, defined and implemented under its responsibility. It includes a set of means, behaviors, procedures and actions adapted to the specific characteristics of each company which

Contributes to the control of its activities, the efficiency of its operations and the efficient use of its resources;

Must allow it to appropriately take into account significant risks, whether operational, financial or compliance. » (Renard, 2012, P5). [2] 


\section{Impact Evaluation of The Internal Control System on The Sales Function Performance Within Moroccan Companies' Context}

After reviewing the main definitions of internal control, it seems important to us to focus on the details of the mechanisms that constitute the pillars of an internal control system. To do this, we will use the work carried out by (Renard, 2012) to try to see the impact of each device (including the influencing factors) on the performance of the commercial function of companies, in order to be able to answer our problem. According to Renard, 2012. P128), [2] an internal control system is grouped into six families of devices, namely:

- Goals ;

- The means ;

- Information systems;

- The organization;

- The procedures ;

- The cheking process.

\section{PERFORMANCE SOCIO-ECONOMIC AND COMMERCIAL PERFORMANCE}

(Salgado, 2013) tried to shed light on the concept of performance and concluded that it is a "suitcase" word in the sense that all of its definitions remain fuzzy and multidimensional. [3]

In this work, we will limit ourselves to the literature review relating to a single dimension of performance. Namely the socioeconomic performance. The latter in turn includes four categories, which are organizational performance, social performance, economic, and financial performance and finally commercial performance that we seek to explain via the families of devices mentioned above.

First, organizational performance tells us about how a business is organized to achieve its goals. (Bocco, 2010). [4]

Second, social performance, as its name suggests, provides information on the nature of social links and relationships within the company and provides information on the company's interest in the social aspect. In this sense, social performance can be measured by the quality of social links between entities, managers and employees. (Marmuse, 1997, cited Bocco, 2010). [4]

Thirdly, economic performance, also called financial, remains, for a large number of managers, the only criterion for evaluating and performing their companies. Indeed, experiences have shown that quite a few cases of failure of certain companies are not essentially due to non-financial performance and that their mastery of activities was behind their failures, thus leading to a drop in the turnover achieved and by therefore a commercial non-performance.

In this wake, the concept of commercial performance has taken a small place in the work of researchers and the majority treats it in its global sense. In this work, we have tried to focus on the literature having focused on this particular type of performance such as (Furrer \& Sudharshan, 2003, Quoted by Bocco2010) [4] and (Bughin, 2006, Quoted by Bocco2010) [4]. In fact, the authors of this work link commercial performance in general to the satisfaction of end customers.

Also, (Croteau et al., 2001) [5], pointed out that the performance of a commercial function can be measured by referring to three dimensions. To know:

- Efficiency (comparison between commercial objectives and achieved results);

- Efficiency (comparison between the resources mobilized and the results of the function);

- Relevance (the comparison between the resources mobilized and the function's objectives).

\section{DEVICES OF CONTROL INTERNAL AND PERFORMANCE TRADE: WHAT RELATIONSHIP?}

According to (Renard, 2012), an internal control system includes six families of devices [2]. First, we will discuss the Objectives family. Indeed, the setting of objectives represents the first reference to which each manager reserves a particular interest. Let's take the example of the commercial function, a team called upon to make sales must refer to minimum thresholds, whether quantitative or qualitative, which make it possible to measure the productivity of the team and to have an idea of the level of commitment and the degree of involvement of each employee in order to be able to decide between them during evaluations. We can argue at this stage that goals give rise to competition and therefore to a mindset of excellence that will undoubtedly lead to a certain performance.

According to March's thesis (1978), the limitations of individuals working in the commercial function are limits of the capacity to calculate the future consequences of their current actions, given the complications of forecasting future consequences, and forecasting decisions related to these consequences, the organization of these decisions and the use of their memories. March (1978) then suggests the development of decision procedures which are reasonable and which take into account the constraints of the function. On this basis, we develop the following hypothesis: 


\section{Impact Evaluation of The Internal Control System on The Sales Function Performance Within Moroccan Companies' Context}

4.1. Hypothesis-1: the absence of objectives could have a negative impact on the performance of the sales function.

(Renard, 2012) also underlines that the 'Objectives' control system, set up within the sales function, will be of no use in cases where:

- The objectives of the commercial function are not known and communicated;

- Risks that could harm the objectives of the sales function have not been identified;

- Failure to set a policy within the sales function that can achieve objectives and manage risks;

- Action plans capable of achieving this policy have not been established.

On this basis, we will extract the four sub-hypotheses linked to our first hypothesis cited above:

Under hypothesis-1.1: Ignorance and non-communication of the objectives set to the commercial functions would negatively influence the performance of the latter.

Under hypothesis-1.2: Failure to identify the risks inherent in the activities of the commercial functions could have a negative impact on the performance of the latter.

Sub-hypothesis-1.3: The absence of a policy to achieve the objectives of the commercial functions would influence the performance of the latter

Sub-hypothesis-1.4: The absence of action plans to deploy the policies of the commercial functions would negatively influence the performance of the latter.

The second family of devices is the Means. It should be emphasized that the setting up of the 'objectives' control mechanism is behind the setting of the means. In this sense, several findings of anomalies issued by internal auditors are mainly due to an insufficiency or inadequacy of the means made available to achieve the objectives set.

On this basis, we can develop our second hypothesis:

\subsection{Hypothesis-2: the lack of resources could have a negative impact on the performance of the sales function.}

In addition, the concept Means encompasses several forms and categories. In previous research, especially empirical research, talking about means often refers to a separation by type of the latter. We cite, for example, human resources, financial resources, technical resources and commercial resources.

In this sense, we can formulate the sub-hypotheses below:

Under hypothesis 2.1: The lack of human resources to achieve the objectives set for the commercial functions would have a negative influence on the performance of the latter.

Sub-hypothesis 2.2: The lack of financial means to achieve the objectives set for the commercial functions would negatively influence the performance of the latter.

Sub-hypothesis 2.3: The absence of technical means to achieve the objectives set for the commercial functions would negatively influence the performance of the latter.

Under hypothesis 2.4: The absence of commercial means to achieve the objectives set for the commercial functions would have a negative influence on the performance of the latter.

Thirdly, we will discuss the most sensitive and most important family of internal control systems, particularly in complex structures. These are Information Systems. Information and communication play a vital role in the success of any coordination and are the essential basis of all decision-making. Information systems provide internal control actors with information that will be assessed and therefore mobilized. For others, they are dispensable in the sense that all of the company's activities are coordinated and monitored through information and communication. Indeed, without these, the internal control system will be doomed to failure, insofar as all those responsible for its implementation will not be informed OUTSEKI J. (2019). Also, the decision based on information extracted from information systems cannot be taken in the absence of said information systems (Rittenberg, Larry E, 2006). [6]

In practice, we cannot speak of the effectiveness of an information system considered as an internal control device within a sales function unless it is capable of fulfilling the following conditions:

- Sales function activity monitoring dashboards are installed.

- Sales function management dashboards are installed.

- Commercial management dashboards are installed.

Despite this, we can say that the efficiency of information systems could guarantee the permanent availability of reliable and useful information that will serve as data channels that will allow operational managers to better control all activities and guarantee the desired performance.

On this basis, our third hypothesis will be: 


\section{Impact Evaluation of The Internal Control System on The Sales Function Performance Within Moroccan Companies'}

Context

4.3. Hypothesis-3: the absence of information systems could have a negative impact on the performance of the commercial function.

As presented above, the variables that can positively or negatively impact the contribution of information systems as an internal control system are monitoring, management and finally steering dashboards. In addition, piloting is the pinnacle of the COSO model (2013). It assumes that the effectiveness of the internal control process is linked to its periodic and / or ad hoc evaluation and to communicate the weaknesses internally of the organization in order to put in place corrective actions (feedback loop) (Noiret and Walter, 2008; Vaassen et al, 2009; Ki-zerbo, 2013, cited by OUASHIL \& OUHADI, 2019). [7]

This is what leads us to develop the three sub-hypotheses below:

Under hypothesis 3.1: The absence of dashboards for monitoring the activities of the commercial functions would have a negative influence on the performance of the latter.

Under hypothesis 3.2: The absence of management dashboards for the activities of the commercial functions would have a negative influence on the performance of the latter.

Under hypothesis 3.3: The absence of management dashboards for the activities of the commercial functions would have a negative influence on the performance of the latter.

The fourth family of internal control systems is the Organization. Fayolle said that we can only control what is organized (Renard, 2012, P.135) [2]. From this quote we can conclude that control is closely related to the organization. Also, according to the same author, a good organization depends on three variables, namely:

- Appropriate and logical organization: for a sales function to be efficient, its organization should be adapted to the types of activity, the environment and especially the culture.

- Objective and reasoned organization: for a sales function to be efficient, its organization should take into account the skills of its resources and place them in positions consistent with their basic training.

Organization based on the distribution of tasks: If a sales manager plans to assign the same person tasks that are incompatible. The latter runs the risk of not controlling all of the activity and therefore giving rise to situations of fraud. Control is a culture based primarily on the separation of tasks in general and incompatible ones in particular.

That said, the organization adopted within the commercial functions would present the essential criterion to hope for a performance of the latter. In this sense, we develop our fourth hypothesis and the three sub-hypotheses that emerge from it:

4.4. Hypothesis-4: the absence of the organization could have a negative impact on the performance of the sales function.

Sub-hypothesis 4.1: The absence of a suitable organization within the sales functions would negatively influence the performance of the latter.

Sub-hypothesis 4.2: The absence of objective organization within the commercial functions would negatively influence the performance of the latter.

Sub-hypothesis 4.3: The absence of organization based on the separation of tasks within the commercial functions would negatively influence the performance of the latter. The fifth family of devices is the Procedures. In fact, all control activity includes standards and procedures deployed within the company to anticipate the risks likely to hinder the achievement of the set objectives (Mandzila, 2004) [8]. An absence of the procedures which outline all the controls to be carried out by the operational staff within all the activities linked to the sales function would result in non-achievement of the objectives and consequently a non-performance of the entire function, hence the hypothesis next :

\subsection{Hypothesis 5: the absence of procedures could have a negative impact on the performance of the sales function.}

As for the sixth and last family of devices, in this case Verification. This system refers to the supervision exercised by the direct hierarchy which is called upon to ensure the level of control of the company's activities. This is a first level control by which operational managers demonstrate to their teams that their actions are verified and monitored. This exercise, carried out at random frequency, promotes compliance with operating procedures, avoids the occurrence of identified risks and would contribute to the performance of the company's functions, including the commercial one. This link will allow us to develop our fifth hypothesis:

\subsection{Hypothesis 5: the absence of verification could have a negative impact on the performance of the sales function.}

In addition, it is important to cite the factors likely to create negligence on the part of those responsible for verification, such as disproportionate trust in the teams, the lack of materialization of the verifications carried out, the incompetence and skill of the actors responsible for the verification. Verification and finally the supervision mode (on site or remotely).

All of these factors will give rise to the sub-hypotheses below: 


\section{Impact Evaluation of The Internal Control System on The Sales Function Performance Within Moroccan Companies' Context}

Sub-hypothesis 5.1: The absence of supervision due to trust in the sales function teams would negatively influence the performance of the latter.

Sub-hypothesis 5.2: The absence of materialization of the verifications carried out within the commercial functions would have a negative influence on the performance of the latter.

Sub-hypothesis 5.3: The lack of competence and skills of the auditors of the activities of the commercial functions would negatively influence the performance of the latter.

Sub-hypothesis 5.4: The mode of verification (on site / remotely) of the activities at the level of the commercial functions would have a negative influence on the performance of the latter.

Finally, on the basis of our literature review, the non-performance of the sales functions would be the result of:

- Ignorance and non-communication of the objectives set for commercial functions.

- The lack of human, financial, technical and commercial resources necessary to achieve the objectives set.

- The lack of an information system making it possible to provide dashboards for monitoring, managing and steering the activities of the sales function.

- The absence of the appropriate and objective organization avoiding any accumulation of incompatible tasks by the same resource.

- Finally, the absence of direct and indirect verification.

After formulating the six hypotheses on the relationships between the different variables of our model, we will graphically design our conceptual model for empirical analysis of data on the performance of the commercial function to try to verify it later with our sample. Of Moroccan companies.

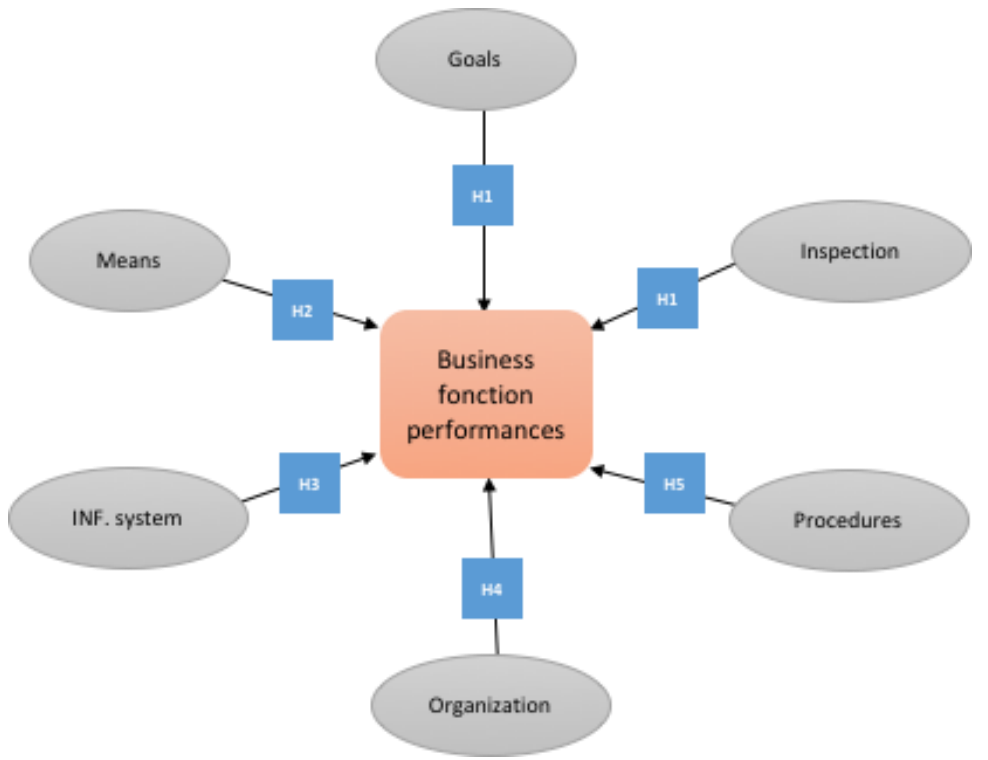

Figure 1: Conceptual research model (RCM).

\section{RESULTS OF THE EMPIRICAL STUDY}

The empirical study carried out focused on a sample of 161 Moroccan companies all having an internal audit structure and commercial departments and we will also find there, specialized audit firms having carried out internal audit missions and of advice. Our objective, via this quantitative study, is to validate the hypotheses made above by using statistical tests and validated measurement scales.

Let us recall our first starting hypothesis relating to the ignorance and non-communication of the objectives set to the commercial functions. The results of unilabiate statistics have shown that a large number of companies in the interviewed sample confirm that the objectives are set and applied to their commercial functions, i.e. (91.8\% of responses). Within the commercial functions of Moroccan companies, the objectives are set and broken down by reference to policies, which guarantee their achievement, and to analyze of the risks, which may hinder their achievements. Despite this, the objectives as an internal control device positively and significantly affect the performance of the sales function within Moroccan companies.

The second hypothesis relates to the absence of the human, financial, technical and commercial resources necessary to achieve the objectives set. The statistical results showed that $89.13 \%$ of the managers of the commercial departments questioned confirm the availability of the human, financial, commercial and technical resources necessary to carry out the operations undertaken at the 


\section{Impact Evaluation of The Internal Control System on The Sales Function Performance Within Moroccan Companies' Context}

level of their various activities. On this basis, we can confirm that in Morocco, the performance of the commercial functions is ensured by the availability of human, financial, commercial and technical resources necessary for the execution of the fixed action plans.

Verification of the third hypothesis relating to the absence of information systems and its impact on the performance of the commercial function revealed the following, based on the answers provided:

- The overwhelming majority of Moroccan companies in the sample studied have information systems through which they publish daily monitoring reports on their activities.

- In Morocco, companies monitor the achievement of their commercial objectives using management dashboards and the implementation of committed action plans using steering dashboards. That is $95 \%$ of our sample.

The fourth hypothesis relating to the absence of the organization as an internal control device and its influence on the performance of the commercial function has been validated and the results obtained confirm it. That said, according to the answers obtained, the majority of managers of commercial departments $(91.6 \%)$ agree that within their commercial functions there is an objective structure (hierarchical organization chart) which takes into consideration the principle of separation of duties. .

As for the procedures and their impact on the performance of the commercial function, the study carried out confirmed that Moroccan companies agree on the evidence that their activities within their commercial functions are governed by written procedures which serve better master the management operations of each activity.

Finally, the impact of the verification system, established within the commercial functions, on the performance of the latter is justified in the sense that a majority represented by a percentage of $89.01 \%$ of Moroccan operational managers confirm that the control operations have been carried out. Provided by direct and indirect managers with traceability and materialization of findings to mitigate any risk that may give rise to poor performance.

\section{CONCLUSION}

Based on our empirical study, the answer to our problematic posed within the framework of this scientific contribution is that the internal control system as presented by its six families of control system, namely the objectives set, the means, information systems, organization, procedures and verification significantly and positively influence the performance of commercial functions. That said, we were able to confirm that the establishment of these systems will guarantee the control of operations within each commercial activity and consequently the effectiveness of the internal control system and the performance of the entire function.

\section{REFERENCES}

1) Economic barometers published by the CGEM https://www.cgem.ma

2) Renard, J. (2012). Comprendre et mettre en œuvre le contrôle interne (1 éd., vol.1, p.13-25). Eyrolles Editions d' Organisation: Paris.

Renard, J. (2010). Théorie et pratique de l'audit interne (7 éd., vol.1, p.13-25). Eyrolles Editions d' Organisation: Paris.

3) Salgado, M. (2013). Analysis of the fundamentals of performance and its valuation.

4) Bocco, BS (2010). Perception of the concept of performance by managers of small businesses in Africa. La Revue des Sciences de Gestion, (1), 117-124.

5) (5) Croteau, AM, RAYMOND, L \& Bergeron, F. (2001). Strategic behavior, choice and management of information systems: Contribution to performance. Information systems and management, 6 (4).

6) OUTSEKI J. (2019) " International Journal of Management Sciences, 2019 - revue-isg.comNumber 3: April 2019 / Volume 2 number 2 "p: 635-650.

7) Ouashil, M. M'bark, and Said Ouhadi. "Le contrôle interne face à l'émergence de nouvelles formes des risques: cas de la fraude." Revue Internationale des Sciences de Gestion (2019): 805-819.

8) Mandzila, Eustache Ebondo Wa. LA CONTRIBUTION DU CONTROLE INTERNE ET DE L'AUDIT AU GOUVERNEMENT D'ENTREPRISE. DisS. UNIVERSITE PARIS XII VAL de MARNE, 2004.

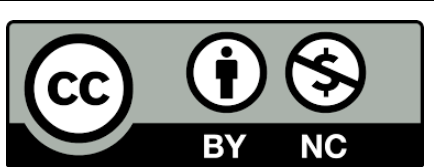

There is an Open Access article, distributed under the terma of the Creative Commons Attribution - Non Commercial 4.0 International (CC BY-NC 4.0)

(https://creativecommons.org/licenses/by-nc/4.0/), which permits remixing, adapting and building upon the work for non-commercial use, provided the original work is properly cited. 